\section{REVIEW ARTICLE}

\title{
Differential regulation of cell wall biogenesis during growth and development in yeast
}

\author{
Gertien J. Smits, $\uparrow$ Herman van den Ende and Frans M. Klis \\ Author for correspondence: Frans M. Klis. Tel: +31 20525 7834. Fax: +31 205257934. \\ e-mail: Klis@bio.uva.nl
}

Swammerdam Institute for Life Sciences, BioCentrum Amsterdam, University of Amsterdam, Kruislaan 318, 1098 SM Amsterdam, The Netherlands

Keywords: cell wall dynamics, glucan, chitin, cell wall proteins

\section{Overview}

The yeast cell wall is a main determinant of cellular strength, and plays an important role in morphogenesis. It is also the first interface with the environment. It has a layered structure, composed mainly of glucan $(60 \%)$, mannoproteins $(40 \%)$ and a small amount of chitin $(1 \%)$ (reviewed by Orlean, 1997). The molecular makeup of the cell wall is known in detail. On the outside of the cell wall, exposed to the environment, are the cell wall mannoproteins (see Fig. 1 for a model of the molecular architecture of the wall). Two classes of covalently linked cell wall proteins can be distinguished. The Pir protein family consists of four members. They are highly $\mathrm{O}$-glycosylated and linked, possibly through their O-glycosyl side chains, to the underlying $1,3-\beta$ glucan network, which provides rigidity to the wall. The other class is that of the GPI-dependent cell wall proteins, almost 40 in number (reviewed by Smits et al., 1999). In addition to $\mathrm{O}$ - and $\mathrm{N}$-glycosylation, they are modified with a GPI-anchor. The GPI-anchor is cleaved off at the plasma membrane, leaving only a sugar remnant, and this sugar remnant is linked to $1,6-\beta$ glucan $(5-10 \%$ of the wall weight), which in turn is bound to $1,3-\beta$-glucan. On the inside of the wall, a small amount of chitin is bound to the 1,3- $\beta$-glucan (reviewed by Kapteyn et al., 1999a).

Cell growth and development demand that the cell wall is not just rigid and unchangeable. In fact, the cell wall changes continuously, during growth, during different developmental programmes such as mating or sporulation, and in response to changes in environmental conditions. Recently, new insights into cell wall biogenesis and adaptation have emerged. Here, we will review these findings and relate them to the cell wall as a dynamic structure.

†Present address: Netherlands Cancer Institute, Department of Cell Biology, Plesmanlaan 121, 1066 CX Amsterdam, The Netherlands.

\section{Cell wall biogenesis during the cell cycle}

During cell division in yeast, cell surface expansion occurs asymmetrically, as shown in Fig. 2. Before bud emergence, secretory vesicles accumulate at the incipient bud site. From the moment of bud emergence, cell wall glucan and mannoproteins are deposited in the bud only, first all over the bud surface, but very soon mainly at the tip of the bud: the apical growth phase. When the bud has reached a size of approximately two-thirds of the mother, buds switch to isotropic growth and cell wall material is deposited over the entire bud surface. After nuclear division is completed, a primary, chitinous septum is generated in the neck region, and a secondary septum is deposited on both sides of the primary septum. The primary septum is degraded by chitinase, separating mother and daughter. Only limited amounts of material are deposited in the mother cell wall during the entire cell division cycle (for a review, see Cabib et al., 1997).

\section{Glucan metabolism}

Regulation of glucan synthesis. Glucan, most of which in yeast is $1,3-\beta$-glucan, gives rigidity to the cell wall. $1,3-\beta$-Glucan is synthesized by the $1,3-\beta$-glucan synthase, which consists of a catalytic and a regulatory subunit. To ensure correct timing and localization of $1,3-\beta$ glucan synthesis during the cell cycle, several mechanisms are employed. First, transcription of FKS1, which encodes the catalytic subunit during growth on glucose, is cell-cycle-dependent, peaking late in G1 phase, shortly before bud emergence (Spellman et al., 1998). Second, and probably more importantly, the activity of the glucan synthase is regulated by the GTPase Rho1p (reviewed by Cabib et al., 1998). Interaction between Fks1p and GTP-bound Rho1p is required for the activity of Fks1p. Rho1p activity is regulated by a set of proteins. Rom1p and Rom2p, which are GDP-GTP exchange factors (GEFs), activate Rho1p (Ozaki et al., 1996), 


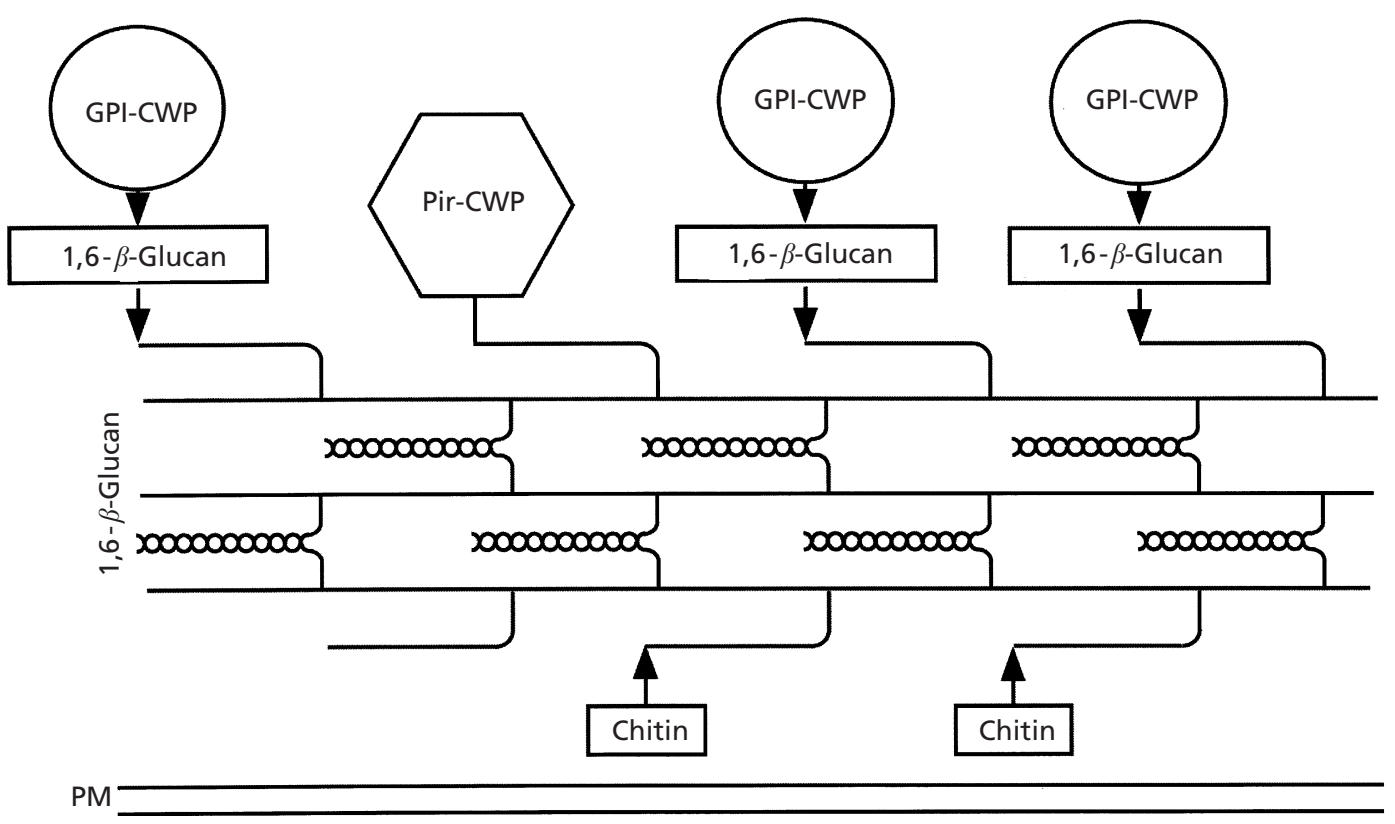

Fig. 1. A model for the molecular architecture of the yeast cell wall. The internal skeletal layer consists of $1,3-\beta$-glucan molecules that form a three-dimensional network surrounding the entire cell. This network is strengthened through multiple hydrogen bridges between aligned 1,3- $\beta$-glucan molecules. At the outside of the skeletal layer, cell wall proteins are linked to the non-reducing ends of 1,3- $\beta$-glucan molecules either directly (Pir-CWPs) or indirectly through an interconnecting 1,6- $\beta$-glucan moiety and the remnant of a GPI-anchor (GPI-CWPs). Some GPI-CWPs, such as Cwp1p, may be linked both ways. After cytokinesis, the skeletal layer becomes strengthened by the coupling of chitin chains to nonreducing ends of 1,3- $\beta$-glucan chains. This takes place mainly at the inside of the skeletal layer. 1,6- $\beta$-Glucan is much more branched than 1,3- $\beta$-glucan (see Klis, 1994). Thus, 1,6- $\beta$-glucan probably functions as a flexible tether for GPI-CWPs. For reasons of clarity, non-covalently bound proteins and proteins linked through disulfide bridges to other cell wall proteins have been omitted. This model is reprinted from Smits et al. (1999) with permission from Elsevier Science.

while SAC7 and BEM2, encoding GTPase-activating proteins (GAPs), decrease Rho1p activity (Schmidt et al., 1997) (see Fig. 3e). Fks1p is an integral membrane protein, and Rho1p can be geranylgeranylated at the C terminus to facilitate association with the plasma membrane. Prenylation, and thus probably membrane localization of Rho1p, is required for $1,3-\beta$-glucan synthase activity, as a mutation in the $\beta$ subunit of yeast geranylgeranyltransferase, $C D C 43$, as well as mutations in Rho1p that abolish prenylation, lead to drastically reduced levels of glucan synthase activity (Inoue et al., 1999). Prenylation of Rho1p thus represents an additional possibility for the control of $1,3-\beta$-glucan synthesis.

Localization of glucan synthesis. Besides regulating the catalytic activity of the glucan synthase, Rho1p is also required for its localization and in general for cellular polarization. Rho1p is localized to active growth sites, as is actin (reviewed by Cabib et al., 1998). For localization of Rho1p, Sla1p, a protein that is important for actin nucleation, is required (Ayscough et al., 1999). Rho1p seems to be unable to polarize in unbudded sla1 mutants, whereas polarization in budded cells seems to be mostly unaffected, indicating that in later cell cycle phases proteins other than Sla1p are responsible for Rho1p polarization. Also, polarization of Rho1p requires a polarized actin cytoskeleton (Ayscough et al., 1999). Rho1p in turn signals to the actin cytoskeleton through Bni1p (Fig. 3a), and is required for actin polarization under multiple conditions (Delley \& Hall, 1999; Drgonová et al., 1999; Helliwell et al., 1998b). Therefore, polarization of Rho1p and the actin cytoskeleton are mutually dependent.

Rho1p function varies during different phases of the cell cycle. Around START, the cell cycle commitment point late in G1 phase, it seems to play an important role in the polarization of the actin cytoskeleton toward the incipient bud site. At this stage, glucan synthesis does not seem to be an essential process, and RHO1 mutants show no significant reduction in glucan synthase activity, although they do show defects in actin polarization (Drgonová et al., 1999). This indicates that late in G1 phase Rho1p only functions in this latter process. In small-budded cells, however, studies with glucan synthase inhibitors revealed that glucan synthesis becomes essential for viability, and Rho1p now becomes essential in its capacity as the regulatory subunit of glucan synthase (Drgonová et al., 1999) (see Fig. 3a, b).

Fks1p itself also localizes to active growth sites (see Cabib et al., 1998), and this localization is dependent on both Rho1p and the actin cytoskeleton (Delley \& Hall, 1999). Fks1p polarization also requires the Slt2-MAP 


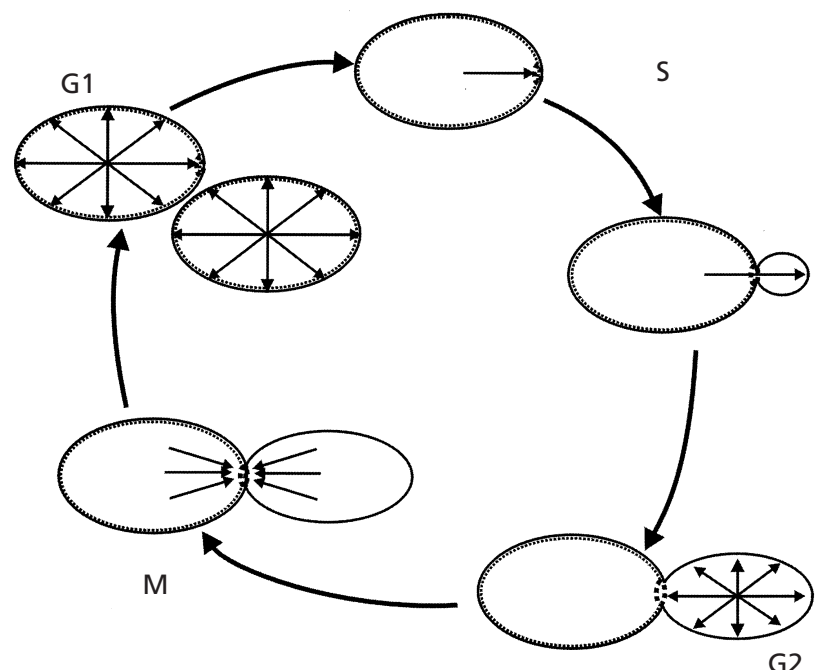

$\mathrm{G} 2$

Fig. 2. Cell wall biosynthesis occurs asymmetrically during the cell cycle. Prior to the initiation of a new cell cycle, a chitin ring forms at the incipient bud site (top). During bud growth, no cell wall material is deposited in the mother cell. Bud growth occurs at the tip (apical growth) until the bud reaches a size of approximately two thirds of the mother, when a switch to isotropic growth is made and cell wall material is deposited all over the bud. During cytokinesis and cell separation, cell wall synthesis takes place in the mother-bud neck, where a three-layered septum is generated. After degradation of the chitinous primary septum, the two individual cells experience cell wall synthesis over their entire surface. Arrows indicate the polarization of the actin cytoskeleton in the depicted stage of the division cycle. Dashed lines indicate the presence of chitin, which in the lateral wall is generally synthesized only after cytokinesis.

kinase pathway (Delley \& Hall, 1999), which can be activated by Rho1p (see Martin et al., 2000, and references therein), and is activated periodically during the cell cycle, particularly during periods of polarized growth (for a review, see Cabib et al., 1998). Pkc1p, which is activated by Rho1p and in turn activates the Slt2-MAP kinase pathway, is also localized to sites of active growth, in a manner dependent on Rho1p and partially dependent on the actin cytoskeleton (Andrews \& Stark, 2000). Unfortunately, the outputs of the Slt2MAP kinase cascade are not fully understood, although it is clear that this cascade is involved in transcriptional regulation of several genes involved in cell wall biosynthesis (Igual et al., 1996; Jung \& Levin, 1999), including an alternative $1,3-\beta$-glucan synthase catalytic subunit, FKS2, which is transcribed in response to cell wall stress (Zhao et al., 1998) as well as during growth on carbon sources other than glucose (Mazur et al., 1995). Most likely, more direct effectors, i.e. without the involvement of transcription, also play an important role in the case of glucan synthesis. Nevertheless, it seems clear that Rho1p and the actin cytoskeleton together provide the spatial cues for directing the glucan synthase catalytic subunit to where it is required, and Rho1p is present at those sites to activate glucan synthesis.
Glucan modification. Whereas, in vitro, 1,3- $\beta$-glucan synthase generates long, linear chains of glucan, the 1,3$\beta$-glucan in the wall is a branched polymer, indicating that the glucan chains are modified during, or shortly after, synthesis. Indeed, a whole set of $1,3-\beta$-glucanmodifying enzymes has been identified. Various (in vitro) glucanase activities can be observed in yeast, caused by glycosylated proteins which are noncovalently linked to the cell wall (Cappellaro et al., 1998) and are also found in the growth medium. The major exoglucanase in vegetatively growing cells is encoded by EXG1, responsible for $90 \%$ of the exoglucanase activity (for a review, see Orlean, 1997). The major endoglucanase is encoded by $B G L 2$. Bgl2p was later shown also to have transglucosylase activity, which is its predominant activity in the presence of relatively high concentrations of glucan non-reducing ends, as is the case in the cell wall (Goldman et al., 1995). In vitro, Bgl2p transfers 1,3- $\beta$-linked oligosaccharides of at least five residues to the non-reducing terminus of another 1,3 - $\beta$-glucan chain, which is at least four residues long, and links them through a $1,6-\beta$-linkage, thereby creating a kink. Possibly, it is involved in connecting newly synthesized $1,3-\beta$-glucan chains to existing ones. Whether actual branches can be created by this enzyme remains to be established.

Another protein involved in modification of the 1,3- $\beta$ glucan is the GPI-anchored plasma membrane protein Gas1p. In vitro, this protein catalyses the hydrolysis of oligosaccharides of different lengths from $1,3-\beta$-glucan chains at least 10 residues long, and the subsequent transfer of the reducing end of the released fragment to the non-reducing end of another glucan chain (Mouyna et al., 2000), thus elongating existing chains. In contrast to $\mathrm{Bgl} 2 \mathrm{p}$, where the new linkage is $1,6-\beta$-glycosidic, Gas1p glucosyl transfer leads to a 1,3- $\beta$-linkage. Therefore, the enzyme likely extends newly formed, short branches, which might after elongation serve as acceptor sites for cell wall proteins or 1,6- $\beta$-glucan chains.

Transcription of GAS1 and EXG1 is cell-cycle-controlled, in a similar manner to FKS1, with mRNA levels peaking in G1 phase (Spellman et al., 1998). Likewise, glucanase activity seems to be regulated in a cell-cycledependent manner. It thus appears that glucan synthesis and subsequent modification are both cell-cycle-controlled, presumably in a coordinated fashion.

\section{Chitin metabolism}

Chitin synthesis and deposition are strictly cell-cycledependent (for a review, see Orlean, 1997). Shortly before budding, chitin is deposited in a ring at the incipient bud site (see Fig. 2). This ring remains in the neck during bud emergence and bud growth, and prevents the neck from widening. During cell separation the ring remains on the mother and becomes the bud scar ring. This ring prevents stretching of the bud scar, in contrast to the birth scar, which is seen on the daughter after cell separation and does not contain a chitin ring, and which becomes much larger during the 
(a)
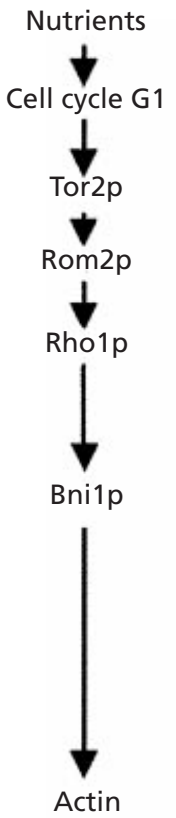

polarization (b)

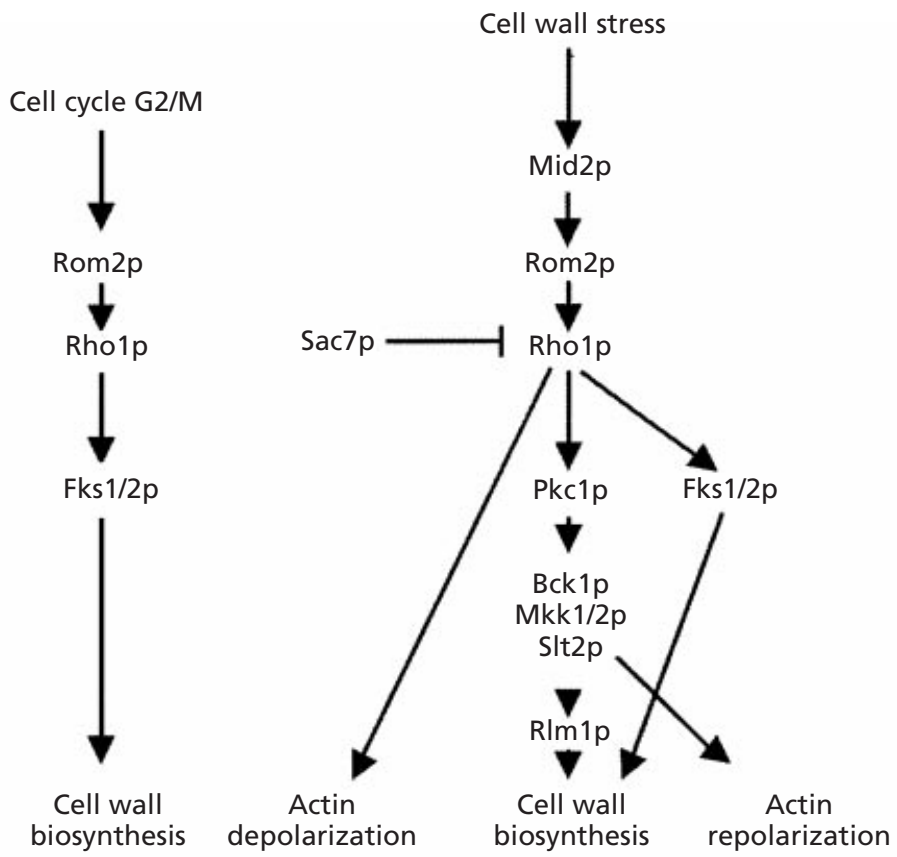

(d)

Oxidative stress?

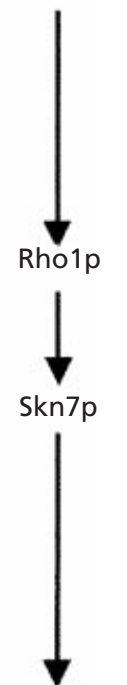

Cell wall biosynthesis

(e)

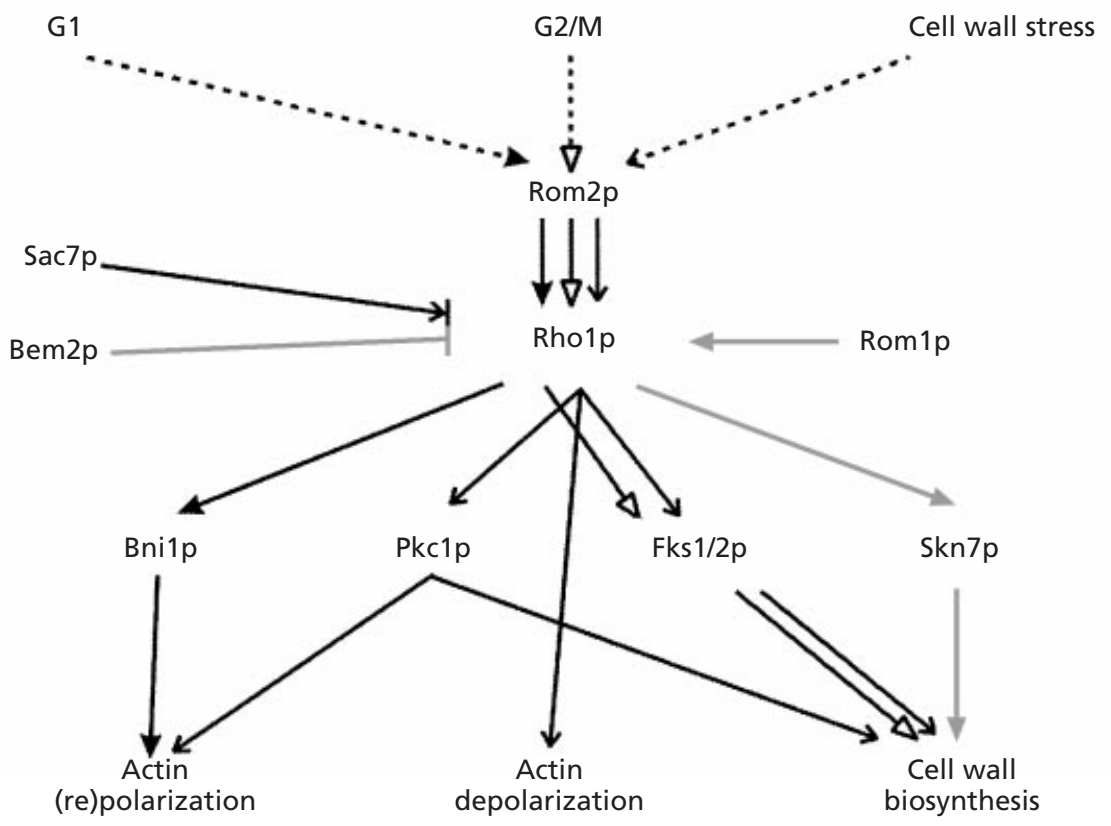

Fig. 3. Cell wall and actin cytoskeleton signalling pathways through Rho1p. Multiple inputs lead to alterations and adaptations in the actin cytoskeleton and cell wall biosynthesis, and many involve Rho1p and Pkc1p. However, different inputs remain separate, and have different outputs. The top figures show complete pathways. (a) In G1 phase, nutrient state is signalled and leads through Tor $2 p$ to polarization of the actin cytoskeleton towards a site for bud initiation. (b) In G2/M phase, glucan synthesis is required for bud growth and the essential function of Rho1p is now activation and localization of the glucan synthase. (c) Cell wall damage activates a combination of responses, including a transient depolarization of the actin cytoskeleton, probably to redirect cell wall biosynthetic enzymes to regions where extra cell wall strength is required. Pkc1p is involved in the repolarization. Cell wall biosynthesis is activated both through direct activation of the enzymes involved (Rho1p activates glucan synthase), and through a transcriptional activation of many genes involved in cell wall biosynthesis through the PKC1-SLT2 MAP kinase pathway. (d) Skn7p is a fourth effector of 
process of cell separation. This asymmetric stretching and the resulting shearing forces probably facilitate the process of cell separation.

Another location of chitin incorporation is the septum. After the bud has grown sufficiently large, the plasma membrane invaginates and a disk of chitin is formed centripetally in the bud neck. Subsequently, material of the secondary septum is deposited on both sides of the disk, thus forming a trilayered septum. After septation, chitin is deposited in the lateral wall of the bud. To generate this rather complex pattern of synthesis and incorporation, three different chitin synthases are secreted to the plasma membrane (Chuang \& Schekman, 1996; Ziman et al., 1996). Their activities are differentially regulated by different and separate mechanisms.

Septum synthesis. The chitin synthase responsible for the deposition of the chitin disk in the septum is CSII, encoded by CHS2 (see Orlean, 1997). CSII activity is strictly cell-cycle-regulated, peaking shortly before cell separation (Choi et al., 1994a). Also, Chs2p, the protein responsible for CSII activity, is localized to the bud neck, but is only present at the end of mitosis, during anaphase and telophase (Chuang \& Schekman, 1996). This strict regulation of activity is dependent on a rapid, cell-cycleregulated synthesis and subsequent (essentially constitutive) vacuolar degradation of the protein (Choi et al., 1994a; Chuang \& Schekman, 1996). What exactly determines the location of the protein remains unknown, although the septins, proteins which constitute the $10 \mathrm{~nm}$ filament ring in the neck, play an important role. In a gin $4 \Delta$ mutant, for example, septation is affected and in many cells chitin is deposited to the sides of the neck without closing the septum, which correlates with the fact that the septins no longer align in a ring but rather in bars in parallel to the mother-bud axis (Longtine $e t$ al., 1998).

The means for specific localization of CSII activity have yet to be established. However, as around the time of cytokinesis the actin cytoskeleton and secretory system are polarized towards the neck region, the restriction of Chs $2 p$ presence to a short period of the cell cycle is probably the main mechanism for confining the protein to the bud neck for a short time at mitosis, and therefore for the localized and temporal synthesis of the chitin destined for the primary septum.

Septum degradation. For cell separation, the chitinous primary septum synthesized by Chs $2 p$ has to be dissolved. Yeast possesses a single endochitinase responsible for this process. The protein is heavily $\mathrm{O}$ glycosylated (see Orlean, 1997) and is present as a noncovalently bound protein in the wall (Cappellaro et al.,
1998) and in the growth medium. Since excessive chitinase activity might perturb cell wall integrity, correct timing and localization of its activity are important. For correct timing of chitinase activity, transcriptional regulation is required. CTS1, the gene encoding chitinase, is transcribed very early in the G1 phase of the cell cycle, an action controlled by the transcription factor Ace2p. Indeed, deletion of Ace $2 p$ leads to defects in cell separation. The fact that in the birth scar, on the daughter side, no chitin remains, whereas in the bud scar a chitin ring is still apparent after the action of the chitinase, suggests that either the chitinase acts mostly from the daughter side, or that properties (such as the accessibility to chitinase) might differ for chitin synthesized by CSII and CSIII (see below), possibly because different proteins are associated with the different chitin structures.

Cell wall repair. A second chitin synthase, CSI, encoded by CHS1, has a function in cell wall repair. As the primary septum is degraded, holes may form in the birth scar of the daughter in the absence of CSI activity (see Orlean, 1997). Although CHS1 mRNA is very unstable, the corresponding protein Chs1p is not, and the in vitro CSI activity is constant during the cell division cycle (Choi et al., 1994a). Chs1p has to be proteolytically cleaved in order to activate CSI, and hardly any activity can be measured in vitro if the extracts have not been treated with proteases (see Orlean, 1997). Probably, proteolytic activation is strictly regulated in time and space. Although endogenous activators of CSI have not been identified, such a regulatory mechanism seems sensible for a protein with repair functions.

The chitin ring and lateral wall chitin. A third chitin synthase, CSIII, is required for synthesis of the chitin ring at the future bud site as well as the chitin in the lateral walls of cells, and therefore for the major part $(90 \%)$ of chitin in yeast (reviewed by Orlean, 1997). The catalytic subunit for CSIII is Chs3p, which, contrary to Chs1p and Chs2p, is metabolically stable (Choi et al., 1994a). Chs3p is the only chitin synthase in yeast whose activity can be measured without proteolytic treatment of the membranes. The protein is activated by proteolytic cleavage in vivo, and therefore the active fraction represents in vivo activity (Choi et al., 1994a). In a chs $4 \Delta$ mutant, chitin content and CSIII activity are drastically reduced, whereas levels of Chs3p are not (Choi et al., 1994b; Trilla et al., 1997). The lowered activity can be compensated for by trypsin treatment of the membranes, restoring the $V_{\max }$ to (trypsin-treated) wild-type levels, showing that in this mutant the protein is present, but a large fraction is no longer activated in vivo (Trilla et al., 1997). Overexpression of CHS4 leads to an increase in

Rho1p, and is probably activated in response to oxidative stress. Skn7p is also involved in cell wall biosynthesis. (e) Inputs and outputs of Rho1p signalling remain separated. The GAPs Sac7p and Bem $2 p$ and the GEFs Rom 1p and Rom $2 p$ might function in maintaining the separation, but spatial and temporal cues can also fulfil a function. Arrows with different tips indicate signalling pathways that remain separated from each other. Light grey arrows indicate that no specific function or pathway has been shown for the corresponding in- or output. 
CSIII activity (Trilla et al., 1997), which can no longer be increased by trypsin treatment (Ono et al., 2000). Sustained interaction with Chs $4 p$ seems to be required for the activity of the activated Chs $3 p$ (Ono et al., 2000). These data suggest that Chs $4 \mathrm{p}$ is required for proteolytic activation of Chs $3 p$ in vivo.

Chs $4 p$ is not only involved in activation of Chs $3 p$, but also appears to play a role in correct localization of Chs $3 \mathrm{p}$. Chs $3 \mathrm{p}$ localizes to a ring in unbudded cells, to the mother side of the neck in small-budded cells, to the neck region of large-budded cells (Chuang \& Schekman, 1996; DeMarini et al., 1997; Santos \& Snyder, 1997), and to punctate internal structures in all cells (Chuang \& Schekman, 1996; Santos \& Snyder, 1997), which correlates with its function in the synthesis of the chitin ring shortly before bud emergence and of the lateral wall chitin after cytokinesis. Correct localization of Chs3p is dependent on the septins (DeMarini et al., 1997), which are located in a ring in the mother-bud neck (for a review, see Longtine et al., 1996), and which are essential to cytokinesis. Localization of Chs $4 p$ to a ring in unbudded and small-budded cells, and to the neck of large-budded cells, is also dependent on the septins, probably through Bni4p, a protein which directly interacts with both Chs4p and one of the septins, Cdc10p (DeMarini et al., 1997). Chs3p and Chs4p are mutually dependent for correct localization (DeMarini et al., 1997). Taken together, these data suggest that Chs $4 p$ is, through Bni4p, responsible for the interaction of the Chs3p-Chs $4 \mathrm{p}$ complex with the septins.

Trafficking of Chs3p, the major chitin synthase. Several other proteins are involved in the secretion and internalization of Chs $3 \mathrm{p}$ and are therefore important for CSIII activity and correct localization of Chs3p. One of these is Chs7p, which is an integral membrane protein of the endoplasmic reticulum (ER) and specifically involved in ER export of Chs3p, but not of Chs1p and Chs2p (Trilla et al., 1999). Overexpression of CHS3 does not lead to an increase in CSIII activity because the excess Chs $3 \mathrm{p}$ is retained in the ER. However, additional overexpression of CHS7 does lead to an increase in CSIII activity (Trilla et al., 1999), suggesting that Chs7p is specifically involved in Chs3p export from the ER and is a limiting factor in this process.

Another protein involved in Chs $3 p$ localization is Chs $5 p$. Mutants deleted for CHS5 lack $80 \%$ of the CSIII activity (Choi et al., 1994b; Santos et al., 1997), and trypsin treatment cannot compensate for this loss (Choi et al., 1994b), suggesting that Chs3p is not present in the plasma membrane (Santos et al., 1997). Indeed, in the absence of Chs5p, Chs3p localizes to punctate internal structures (Chuang \& Schekman, 1996; Santos \& Snyder, 1997) but no longer to a ring at the incipient bud site or in the neck (Santos \& Snyder, 1997). Chs5p itself localizes to similar spots, which appear to be late Golgi vesicles (Santos \& Snyder, 1997). Nearly all Chs3pcontaining vesicles also contain Chs5p (Santos \& Snyder, 1997). Together, these data suggest a role for Chs $5 p$ in delivery of Chs3p to the correct location in the plasma membrane.
For localization of Chs3p, not only transport to the plasma membrane is important; recycling of the protein through an internalization step also seems to be involved. Subcellular fractionation indicates that Chs1p and Chs $3 p$ are present both in the plasma membrane and in a vesicle compartment called the chitosome (Ziman et al., 1996, 1998). In cells defective in endocytosis, Chs1p and Chs3p accumulate in the plasma membrane and Chs3p no longer localizes to the cytosolic spots (Chuang \& Schekman, 1996; Ziman et al., 1996) that probably correspond to the chitosomes. Chs $2 p$ trafficking is not affected in these mutants (Chuang \& Schekman, 1996). These data show that the chitosomes are, at least partially, derived from endocytic material. The question remains whether chitosomes are exclusively endocytically derived or if they can also mature from late secretory vesicles. In chs6 $\Delta$ mutants, newly synthesized Chs $3 \mathrm{p}$ accumulates in chitosomes that are not endocytically derived since they also accumulate in endocytosis-deficient mutants (Ziman et al., 1996, 1998). Also, the accumulated Chs $3 \mathrm{p}$ is active, showing that it has matured further than in chs $5 \Delta$ mutants, where it cannot be activated, and showing that chitosome characteristics are present before an endocytic step has occurred. Whether the properties of endocytically derived chitosomes are similar to the matured ones remains to be established, but a likely explanation for the accumulated data are the existence of a reversible process where chitosomes can associate with and dissociate from the plasma membrane (or recruit material from there) so that a block in either step leads to the accumulation of chitin synthase in chitosomes or plasma membrane, respectively. The fact that the metabolically stable Chs3p is localized in a ring in unbudded, small-budded and large-budded cells, but not in medium-budded (one-nucleus) cells (Chuang \& Schekman, 1996; Santos \& Snyder, 1997) may satisfactorily be explained by the existence of such a redistribution mechanism for Chs $3 p$. In view of the fact that Chs1p trafficking is not affected in chs $6 \Delta$ mutants, although the protein is found in chitosomes and accumulates in the plasma membrane in an endocytosisdefective mutant (Ziman et al., 1996), a different mechanism must exist for its redistribution. That such a mechanism exists is highly likely, considering the repair function that Chs1p is believed to fulfil.

\section{Cell wall mannoproteins}

The yeast genome encodes approximately 40 different GPI-dependent and 4 Pir cell wall mannoproteins, which are all covalently bound to 1,3- $\beta$-glucan (see Smits et al., 1999). More than $50 \%$ of the genes encoding these cell wall proteins are transcribed in a cell-cycle-dependent manner (Spellman et al., 1998), and most of these are transcribed in $\mathrm{M} / \mathrm{G} 1$ phase. Although the expression of many cell wall proteins is cell-cycle-dependent, their role in the cell division cycle is largely unknown. Egt $2 p$ is the only cell wall protein for which a role in cell cycle progression is known. EGT2 was identified as an early G1 transcript, and its transcription is regulated by Swi5p 
(Kovacech et al., 1996), which is active at the same time as Ace2p, the transcription factor that regulates chitinase expression. Deletion of EGT2 leads to a delay in cell separation, indicating an involvement of this GPIdependent cell wall protein in the regulation of the degradation of the primary septum.

Several cell wall proteins are found in regions of the wall that correlate with the polarization of the secretory pathway around their time of expression (RodriguezPeña et al., 2000; G. J. Smits, unpublished results). For two of these proteins, Tip1p and Cwp2p, the timing of transcription seems to determine the incorporation pattern. A third cell wall protein, Cwp1p, is localized to the birth scar. For this to happen, correct timing of transcription is important but is not the only determining factor. Localization of Cwp1p seems also to be dependent on the presence and correct localization of the chitin in the septum, synthesized by Chs $2 p$. For none of these proteins, however, could a role in cell cycle progression be observed (G. J. Smits, unpublished data). Crh1p and Crh2p localize to chitin-rich areas in the wall (Rodriguez-Peña et al., 2000). These proteins show homology to glycosidases, and the conserved glycosidase domains are required for their function in conferring resistance to cells with respect to lytic enzymes. This suggests they have a role in cell wall construction, possibly with specific functions during the cell cycle (Rodriguez-Peña et al., 2000).

Traditionally, cell wall proteins have been studied in view of their function in cell wall integrity and resistance to lytic enzymes and cell-wall-perturbing agents. The findings that cell wall protein expression is strictly controlled and that the proteins are often localized within specific regions of the cell wall point towards other possibilities. It would be interesting to assess their role in the establishment of polarity and cellular morphology.

\section{Coupling of cell wall macromolecules}

Although the linkages between the different cell wall components have been described in detail, the coupling enzymes involved in the generation of these links have not been identified. The coupling of chitin to $1,3-\beta$ glucan seems to occur in a cell-cycle-dependent manner. Two chemically separable pools of $\beta$-1,3-glucan exist in the wall: alkali-soluble and alkali-insoluble glucan. The alkali-soluble fraction was shown by pulse-chase analysis to be a precursor to the alkali-insoluble fraction, and the insolubility depends on the binding of chitin to the glucan (see Klis, 1994). The two different pools of glucan both increase in a cell-cycle-dependent manner, but whereas the amount of alkali-soluble glucan increases most during bud formation, in agreement with the presumed glucan synthase activity in the bud, the alkaliinsoluble glucan only increases around the time of cell separation, concurrent with chitin incorporation into the lateral wall (reviewed by Klis, 1994). It is to be expected that enzymes exist involved in the cleavage of the GPI-anchor of the GPI-proteins and the subsequent attachment to 1,6- $\beta$-glucan, as well as enzymes involved in coupling $1,6-\beta$-glucan to $1,3-\beta$-glucan, and the coupling of chitin to both glucans. Also, it would be likely that these enzymes act in concert so that, for example, released proteins are kept in the wall and are not washed out into the growth medium.

\section{Cell wall biogenesis during specific developmental programmes \\ Mating}

Haploid yeast cells constitutively secrete mating pheromones. When cells detect pheromones of the opposite mating type, the pheromone response MAP kinase pathway is activated through a trimeric G-protein (reviewed by Gustin et al., 1998), cells arrest cell division in G1 phase, and form a mating projection through the polarization of the actin cytoskeleton and the secretory system in the direction of increasing pheromone concentrations. Such cells are called 'shmoos'. After contact is made through complementary adhesion proteins in the cell wall ('agglutination'), vesicles accumulate close to the contact zone, the walls in this zone are degraded, and the plasma membranes and eventually their nuclei fuse to form diploid zygotes. The zygotes can then start cell division and continue vegetative growth (reviewed by Marsh \& Rose, 1997).

During the formation of the mating projection the cell wall changes. For sexual agglutination, three specific cell wall proteins are required. MAT $\alpha$ cells express the GPIdependent cell wall protein Sag1p, and MATa cells express the binding subunit Aga2p, which is linked via a disulfide bridge to the GPI-dependent anchor subunit Aga1p. Sag1p and Aga2p become highly expressed when the cells are exposed to mating pheromones, and are deposited mostly in the shmoo tip (for a review, see Orlean, 1997). The two proteins interact in a highly specific manner, resulting in very strong binding of the cells. The amount of chitin in the cell wall of the mating projection is drastically increased, and instead of a welldefined ring a diffuse ring is seen at the base of the mating projection (see Orlean, 1997). Responsible for this deposition of chitin is Chs3p, the chitin synthase involved in synthesis of the chitin ring and the chitin in the lateral wall, as well as the chitin synthesis in response to cell wall damage (Osmond et al., 1999; Valdivieso et al., 2000; Garcia-Rodriguez et al., 2000). However, neither Chs3p nor Chs4p is essential for cell fusion. Interestingly, chs $5 \Delta$ mutants do have cell fusion defects (Santos et al., 1997). Chs5p is involved in correct localization of Chs $3 p$, but possibly also in trafficking of other proteins that have a role in fusion. The increased chitin deposition during shmoo formation seems to be a response to the weakening of the shmoo cell wall (see below), since shmooing and mating cells require the cell wall stress sensor Mid2p and the PKC1-SLT2 cell wall integrity pathway for survival (Ono et al., 1994; see Gustin et al., 1998).

Several mutants have been identified that are defective in fusion, designated fus mutants. The vesicles that nor- 
mally accumulate in a highly polarized fashion at regions of cell wall thinning, which takes place at the zone of fusion, are often dispersed in these mutants (Gammie et al., 1998). In many cases, however, the FUS genes do not seem to be directly involved in the cell wall remodelling required for fusion. FUS3, for example, encodes the MAP kinase in the mating signal transduction pathway. An interesting candidate for direct involvement in cell wall remodelling is FUS1, encoding a glycosylated transmembrane protein, the $\mathrm{N}$ terminus of which extends outside the plasma membrane (see Marsh \& Rose, 1997).

For the degradation of the wall in the fusion region, cellwall-degrading enzymes are expected to be delivered there, which might explain the vesicle accumulation. Indeed, a family of secreted glucanases, with homology to the glycosyl transferase $\mathrm{Bgl} 2 \mathrm{p}$, has been identified, two members of which are important for mating (Cappellaro et al., 1998). Yeast mutated for both scw 4 and scw10 shmoo and agglutinate normally, but the prezygotes do not fuse.

Although cell wall proteins are generally important for agglutination and fusion, one GPI-dependent cell wall protein has a specific role in zygote formation. It was first identified based on its increased transcription in cells exposed to high concentrations of mating pheromone and was named Fig2p. Deletion of the gene does not lead to decreased, but rather to slightly increased, agglutination. However, after the cell wall barrier between the haploids has been dissolved and the membranes are fused, karyogamy is not possible because the bridge between the two haploids is too narrow to allow nuclear migration (Erdman et al., 1998). This indicates that cell wall proteins can serve specific roles in cellular morphology, in this case determining the width of the neck.

\section{Pseudohyphal growth}

In diploid yeast, the lack of suitable nitrogen sources in the presence of a sufficient carbon source leads to the induction of pseudohyphal growth. During pseudohyphal growth, cells become elongated and stay attached to the mother, the budding pattern changes to one where cells bud exclusively from their distal pole, cell division of the daughter becomes synchronized with that of the mother because the G2 phase is elongated, and cells acquire the ability to invade solid growth media (see Gustin et al., 1998, Banuett, 1998). On plates, this leads to extended, frayed colonies that also grow below the surface of the agar, and the combined changes are suggested to facilitate foraging for suitable nutrients. Haploid yeast shows a similar growth type after prolonged incubation on rich medium plates. The cells form chains and invade the agar on the edges of the colony, but they do not become elongated. This growth type is referred to as haploid invasive growth (see Gustin et al., 1998).
For the opportunistic pathogen Candida albicans, the ability to infect a host depends on the ability to grow (pseudo)hyphally (Lo et al., 1997). For this organism evidence has accumulated that the walls of the hyphal and yeast forms differ. Differences in antigenicity, and later differences in chitin levels, cell wall composition and protein incorporation, have been observed (see Chaffin et al., 1998, and references therein; Kapteyn et al., 2000). Based on these observations, one may expect similar differences between yeast and pseudohyphal cells in Saccharomyces cerevisiae.

More than one signal transduction pathway is involved in signalling for pseudohyphal growth. A MAP kinase pathway (the filamentous growth pathway) sharing components with the mating MAP kinase cascade is crucial (reviewed by Gustin et al., 1998; Banuett, 1998), but another input is through a G-protein to Ras2p, relaying a signal via PKA (Kubler et al., 1997; Lorenz \& Heitman, 1997; Pan \& Heitman, 1999; Robertson \& Fink, 1998). These pathways are interconnected, since, for example, Ras2p can activate the filamentous growth MAP kinase cascade (Mosch et al., 1996), but they also have separate outputs, as signalling through PKA does not require the MAP kinase (Pan \& Heitman, 1999).

Both signalling pathways converge on a common important output (Rupp et al., 1999), the GPI-dependent cell wall protein Flo11p, which plays a central role in pseudohyphal growth, and the presence of which is sufficient to induce invasion and frayed colony morphology even in the absence of signalling (Lambrechts et al., 1996; Lo \& Dranginis, 1998). Yeast mutated for FLO11, which in diploids is transcribed only when nitrogen is limiting, can no longer invade solid media and colonies lose their frayed edges, suggesting that cell elongation or budding pattern might also be affected. Interestingly, in a diploid strain deleted for SFL1, a transcription factor involved in pseudohyphal growth signalling, some agar invasion and branched colony shape can occur in the absence of Flo11p, suggesting that this protein, although important, is not the only factor that can generate these phenotypes (Robertson \& Fink, 1998).

The fact that mothers and daughters stay attached in the pseudohyphal mode of growth is another important factor contributing to the ability to invade agar. If transcription of CTS1, encoding the chitinase involved in degradation of the primary septum, is abolished, either by deleting the gene or by deleting the transcription factor ACE2, cells invade the agar (King \& Butler, 1998). Indeed, in yeast strains that have the ability to grow pseudohyphally, chitinase activities are much lower than in strains that cannot (King \& Butler, 1998). In conclusion, we can say that the cell wall is crucial to pseudohyphal growth. The proteins incorporated in the wall and cell wall chitin metabolism are important determinants for the ability to invade agar, one of the most striking features of pseudohyphally growing cells. 


\section{Sporulation}

Upon starvation for both carbon and nitrogen sources, diploid yeast cells enter meiosis to generate four haploid spores (for a review, see Kupiec et al., 1997). These spores are formed within the old cell, which now becomes the ascus, and are surrounded by their own ascospore walls. These walls are very different from walls of vegetatively growing cells and are designed to resist severe environmental stresses. The inner layer of the spore wall is composed mainly of glucan and mannoproteins, similar to vegetative cell walls. The inner layer is surrounded by a layer of chitosan, which is deacetylated chitin and thus resistant to the action of chitinases. On the outside of the spore wall is a proteinaceous layer which is made highly insoluble through dityrosine cross-links, a sporulation-specific compound of $\mathrm{D}$ - and L-tyrosine residues covalently linked through their phenyl side-chains, and which amounts to $50 \%$ of the outer layer polypeptide. Together, these layers render the spores resistant to, amongst others, Glusulase and Zymolyase, heat stress and ether (see Kupiec et al., 1997).

Although the inner two layers appear to have a general composition similar to that of the vegetative wall, the details are different. For example, Fks2p appears to be the glucan synthase used during sporulation (Mazur et al., 1995), and micro-array studies suggest that the cell wall protein population differs, with all the genes for the Pir proteins downregulated, as well as SED1, SPI1, CRH1 and CRH2, and genes such as TIR2 and YOR214c upregulated. Several members of the glycosyl transferase GAS family are downregulated to make place for others, and almost all genes in the BGL family are downregulated, whereas two sporulation-specific glucanases become expressed (Chu et al., 1998, and references therein).

For biosynthesis of the chitosan layer, chitin synthesized by Chs $3 p$ serves as a precursor. This is then deacetylated by two different sporulation-specific chitin deacetylases, Cda1p and Cda2p (Christodoulidou et al., 1999). Whereas deletion of CDA2 leads to the absence of the chitosan as well as the dityrosine layer, deletion of CDA1 causes the absence of the dityrosine layer but leaves most of the chitosan layer intact. The formation of the dityrosine layer is described by Orlean (1997).

Sporulation is a process of multiple phases, recognizable not only by the different meiotic phases and progression of spore morphogenesis, but also by a phased transcriptional programme (Chu et al., 1998). Spore wall biosynthesis also appears to be of a sequential nature. For example, the dityrosine layer is not deposited if the chitosan layer is absent or severely defective, as in glucosamine auxotrophs (Wagner et al., 1999), and spore walls missing both outer layers have been observed (Wagner et al., 1999); however spores containing the two outer layers but missing the inner layers have never been described.

Spore wall morphogenesis is controlled by the MAP kinase Smk1p, with Sps1p as a potential upstream activator (reviewed by Gustin et al., 1998). Other members of the MAP kinase module have not yet been identified. Interestingly, subsequent phases in spore wall biogenesis appear to require increasing SMK1 activities (Wagner et al., 1999). For the completion of the glucanrich inner layers less SMK1 activity is required than for the chitosan layer, and the completion of the dityrosine layer requires additional SMK1 activity.

\section{Cell wall biogenesis in response to cell wall stress}

\section{The response to stress signals}

Many organisms have devised mechanisms to defend themselves against fungal invasion. Many of these mechanisms target the cell wall, since it is an organelle essential to the fungus and sufficiently different from that of plants or other hosts. Yeast has developed a mechanism to compensate for these attacks. This response mechanism, often called the cell wall integrity pathway, can be activated in various ways. It is activated in response to glucanases or other cell wall lytic enzymes (de Nobel et al., 2000) in response to cell-wallweakening mutations (Bickle et al., 1998) or hypoosmotic stress, but also, and probably less specifically, to heat stress (see Gustin et al., 1998; Banuett, 1998). Three main responses can be distinguished. First, the synthesis of several cell wall components is increased. $1,3-\beta$-Glucan synthesis is upregulated by the transcriptional activation of the alternative glucan synthase FKS2 (Zhao et al., 1998), and probably also more directly through activation of the regulatory subunit Rho1p (Bickle et al., 1998). Chitin synthesis is drastically increased, and the chitin content of cell walls, normally 1-2\%, can increase to over $20 \%$ (reviewed by Smits et al., 1999). The transcription and incorporation of several cell wall proteins increases (de Nobel et al., 2000; Jung \& Levin, 1999; Kapteyn et al., 1999b), probably to protect the glucan layer against lytic enzymes. Second, the cell switches to alternative mechanisms for incorporation of cell wall proteins. When $1,3-\beta$-glucan in the wall decreases, GPI-dependent cell wall proteins become linked through a direct binding of 1,6 - $\beta$-glucan to chitin, which is consistent with the increased chitin availability (see Kapteyn et al., 1999a). If 1,6- $\beta$-glucan is deficient, the amount of 1,6- $\beta$-glucanase-resistant mannoproteins increases, suggesting that a larger fraction of cell wall proteins becomes incorporated by binding directly to 1,3- $\beta$-glucan and chitin (Kapteyn et al., 1999b), probably because the levels of Pir proteins increase. The third response ensures that cell wall strengthening can occur where weakening takes place. Whereas the actin cytoskeleton, $\mathrm{Pkc1p}$ and the glucan synthase are normally focused to active growth regions, upon cell wall stress (induced by SDS or Zymolyase) they are redistributed all over the cell (Delley \& Hall, 1999; Andrews \& Stark, 2000). This depolarization is transient, and after a while both the actin cytoskeleton and the glucan synthase repolarize. Chs1p and/or Chs3p, the chitin synthases functioning in cell wall 
repair and in chitin synthesis in the lateral wall, might also be redistributed, since they can both be relocalized to chitosomes after an endocytic step (Chuang \& Schekman, 1996; Ziman et al., 1996) and can be redirected to a new plasma membrane location (GarciaRodriguez et al., 2000).

Presumably, the cell does not respond to different cell wall defects in a specific manner, but rather senses cell wall weakening as such and activates all processes that could help maintain cell wall integrity. Also, the net result of these responses is that the cells become resistant not only to glucanases and other cell-wall-disturbing agents, but also to heat stress, showing that the different input signals leading to activation of the response mechanism all lead to a strengthened wall, which is important for tolerance to various forms of stress (de Nobel et al., 2000).

\section{Detection of stress signals and signal transduction}

In the past couple of years, the signal transduction pathway leading to cell wall stress-induced responses has been largely elucidated. The role of the PKC1-SLT2 signalling module (see Gustin et al., 1998; Banuett, 1998) is well established. This module was shown to be involved in heat shock response, osmotic stability and cell wall biosynthesis (Igual et al., 1996). Since the phenotypes of strains mutated for the components of the MAP-kinase module downstream of Pkc1p are less severe than those of $p k c 1 \Delta$, other downstream effectors of $\mathrm{Pkc1p}$ must exist, but these have not yet been identified.

Cell wall defects are sensed in the plasma membrane, possibly in a way that involves membrane stretch (see Gustin et al., 1998; Banuett, 1998). A set of single-span integral membrane proteins, Hcs77p, Wsc2p, Wsc3p, Wsc4p and Mid2p, are the probable sensors (Gray et al., 1997; Jacoby et al., 1998; Ketela et al., 1999; Rajavel et al., 1999; Verna et al., 1997). Although deletion of WSC2 and WSC3 enhances phenotypes of an hcs $77 \Delta$ mutant (Verna et al., 1997), and mid2 $\Delta$ and hcs $77 \Delta$ are synthetically lethal (Ketela et al., 1999; Rajavel et al., 1999), a direct role in the response to cell wall damage has only been shown for Mid2p (de Nobel et al., 2000; Ketela et al., 1999; Martin et al., 2000). High temperature and cell-wall-perturbing agents induce dual phosphorylation of Slt $2 p$ in a mechanism dependent on Mid2p but not on Hcs77p (de Nobel et al., 2000; Martin et al., 2000). On the other hand, depolarization of the actin cytoskeleton in response to high temperature is dependent on Hcs77p, although not completely (Delley \& Hall, 1999).

Downstream of the sensor, Rho1p is activated by cell wall stress (Bickle et al., 1998) through the activation of the exchange factor Rom $2 p$ (Fig. 3c). Rho1p can directly activate glucan synthesis through interaction with Fks1p or Fks2p, but it can also bind, activate and localize Pkc1p and so (locally) activate Slt2p (reviewed by Cabib et al., 1998). Activation of Slt $2 \mathrm{p}$ is transient, and deactivation is dependent on the Rho1p-GAP Sac7p (Martin et al., 2000). Interestingly, although depolarization of the actin cytoskeleton and Fks1p after heat shock and cell wall stress are not dependent on Pkc1p, the repolarization that takes place after approximately $2 \mathrm{~h}$ is dependent on Pkc1p and Slt $2 \mathrm{p}$, showing that the PKC1-SLT2 pathway is required for the reinitiation of growth. The depolarization reaction is also activated in response to overactivation of Rho1p or Pkc1p, but not by downstream components of the PKC-SLT2 pathway, suggesting that other effectors of Rho1p or Pkc1p are involved (Delley \& Hall, 1999) (see Fig. 3c).

MAP kinase cascades often activate transcription factors. A transcription factor downstream of the Slt2MAP kinase module is R $1 \mathrm{~m} 1 \mathrm{p}$ (Dodou \& Treisman, 1997; Jung \& Levin, 1999; Watanabe et al., 1997), but it is not the only one involved in the transcriptional response to cell wall stress. Deletion of RLM1 does not lead to defects in cell wall integrity (Watanabe et al., 1997), and, more importantly, binding sites for $R \operatorname{lm} 1 \mathrm{p}$ in the promoter of FKS2, the best-defined target of the PKC1-SLT2 pathway, are present and involved in transcription activation (Zhao et al., 1998), but the region of the FKS2 promoter responsible for heat shock activation of the gene through the PKC1-SLT2 pathway is separate from that containing the Rlm1p-binding sites. This suggests that another, currently unknown, transcription factor might be involved (Zhao et al., 1998). Surprisingly, transcriptional activation of all other genes induced by overactive Mkk1p, the MAP kinase kinase in the PKC1-SLT2 pathway, was abolished in a $r \operatorname{lm} 1 \Delta$ mutant (Jung \& Levin, 1999), indicating that $F K S 2$ is an exceptional case, although crucial to the cell wall stress response. Possibly, cooperation of R $\operatorname{lm} 1 \mathrm{p}$ with other factors that bind the promoter is required, as is the case for Ste12p, another transcription factor downstream of MAP kinase pathways.

Other cell-wall-related genes of which transcription is activated by Pkc1p are FKS1, GAS1, BGL2 and CHS3, as well as several genes encoding cell wall proteins such as CWP1, SSR1 and several Pir proteins (Igual et al., 1996; Jung \& Levin, 1999). The first three indicate that glucan synthesis and modification are changed in response to cell wall stress, which could lead to altered rigidity of the wall, but could also function in altering the type of linkage used to incorporate proteins in the wall. The cell wall proteins of which transcription is activated are indeed the proteins that become more prominent in the wall in response to cell wall stress (Kapteyn et al., 1999b) including the Pir proteins, which can explain the increase in 1,6- $\beta$-glucanase-resistant proteins. The increased transcription of CHS3 indicates that CSIII is the chitin synthase responsible for the increase in chitin content in stressed cells. This is corroborated by the increase of CSIII activity in $f k s 1$ mutants (Garcia-Rodriguez et al., 2000), and by the dramatically decreased chitin levels in chs3 gas1 mutants as compared to gas1 mutants (Valdivieso et al., 2000). The observation that the Calcofluor white hyper- 
sensitivity caused by overexpression of MID2 is completely relieved in a chs $3 \Delta$ mutant (Ketela et al., 1999) shows that the increased CSIII activity is indeed signalled by this putative cell wall stress sensor.

There are three major unsolved questions regarding the cell wall damage response pathway. First, there is the problem of the sensing of cell wall defects. Not only are the identities and specificities of the putative candidates largely unknown, but also the nature of the signal is currently ill understood. The conformational change of a sensor in the plasma membrane seems an interesting option, as this could occur under most circumstances during which the cell wall integrity pathway is activated (hypo-osmotic conditions, SDS, cell wall weakening caused by mutations or cell-wall-damaging agents), but single-span plasma membrane proteins do not seem good candidates in such a model. Second, it is clear that the MAP kinase module is not the only output of Pkc1p activation, and the other outputs are involved in cell wall integrity but remain largely unknown. They have to do with the depolarization of growth and possibly also with aspects of cell wall reorganization that have not been extensively discussed, such as the changes in linking the different cell wall components. Possibly, the synthesis and structure of 1,6- $\beta$-glucan are also affected.

The last question, which is of more general interest, is how different inputs, through different sensors, can lead to different outputs even if they are relayed through the same pathway. In this regard, Rho1p is particularly enigmatic. It has at least four different effectors, Bni1p (Fig. 3a), glucan synthase (Fig. 3b), Pkc1p (Fig. 3c) and the yeast response regulator Skn7p (Fig. 3d) (see Fig. 3e) (Alberts et al., 1998; Kohno et al., 1996; Cabib et al., 1998), that do not all have to be activated at the same time and place. Rho1p is, through Rom $2 \mathrm{p}$, activated by the phosphatidylinositol kinase homologue Tor $2 p$ (Schmidt et al., 1997). Tor2p is involved in the regulation of translation initiation and through that in cell cycle progression in G1, probably in response to nutritional conditions (Barbet et al., 1996; Di Como \& Arndt, 1996) (Fig. 3a). Also, it has a separate function in the organization of the actin cytoskeleton in G2/M phase (Helliwell et al., 1998a; Schmidt et al., 1996). This last function requires Rho1p and Rom $2 \mathrm{p}$ (Helliwell et al., 1998a; Schmidt et al., 1997), the Rho1p effector Pkc1p and the downstream MAP kinase module, but not Bni1p, Fks1p or Skn7p (Helliwell et al., 1998b) (Fig. 3b). Furthermore, Rho1p has different functions during different phases of the cell cycle, as described above: it is required for the polarization of the actin cytoskeleton in G1, and for glucan synthase activation during bud growth (Drgonová et al., 1999). Since $p k c 1$ mutants have not been shown to have actin polarization defects, Pkc1p is not required for the cell-cycle-regulated polarity of the actin cytoskeleton, and the role of Rho1p in G1 phase polarization probably does not require $\mathrm{Pkc1p}$. The likely candidate would then be Bnilp, which interacts with profilin and affects the actin cytoskeleton of unbudded cells (Imamura et al., 1997; Kohno et al.,
1996) (Fig. 3a). The signal for activation might be Tor2p, required for cell cycle progression in G1 phase.

During bud emergence and growth, Rho1p might primarily activate Fks1p. Its role in activation of the cell wall stress response through Pkc1p should take place only when the cell is compromised (Fig. 3b, c), this also explains why Mpk1p phosphorylation in response to heat shock is not altered in a tor $2^{\text {ts }}$ mutant, which has no detectable cell wall defects (Helliwell et al., 1998b) (Fig. $3 \mathrm{a}, \mathrm{c})$. It is also interesting to note that in an $h c s 77 \Delta$ mutant, cell division control and nutrient response seem affected (Ivanovska \& Rose, 2000), whereas this plasma membrane protein is thought to be involved in cell wall damage sensing. Possibly, loss of components of the signalling pathway leads to a loss of specificity of the responses.

Similar specificities in responses to different input signals have been described for other small GTPases (Reif et al., 1996). Specificity might be generated through specific localization, or through interaction with different cofactors, and both these possibilities might be temporally or spatially regulated. For both functions requiring activity towards the actin cytoskeleton, the exchange factor Rom2p is required (Bickle et al., 1998; Schmidt $e t$ al., 1997) (Fig. 3e). However, there is at least one other exchange factor, Rom1p, that can activate Rho1p, and no specific role for that is yet known (Ozaki et al., 1996). Also, Sac7p, the GTPase-activating protein for Rho1p (Schmidt et al., 1997), functions downstream of Tor2p, and inactivates the Pkc1p pathway (Martin et al., 2000; Schmidt et al., 1997), but a second GAP, Bem2p, involved in bud emergence, is also present without a specific role (Kim et al., 1994) (Fig. 3e). This, and other as yet unidentified factors, may help solve the problem of how one protein can mediate specific responses to specific inputs.

\section{Perspectives}

The cell wall of yeast has been the object of study for many years, but it was considered to be of limited interest to cell biologists. Much was known biochemically, but only recently, with the use of specific enzymes and advanced chemical techniques, has the architecture of the cell wall been determined in detail. Since the cloning of the first glucan synthase by multiple groups and the discovery of the involvement of the molecular switch protein Rho1p in its regulation, the dynamic nature of the rigid wall has become evident. Also, the completion of the sequencing of the yeast genome and the subsequent accumulation of wholegenome transcriptional data has provided new clues for elucidating the functions of many genes with homology to known genes encoding cell wall biosynthetic enzymes. New cell biological techniques, such as live-imaging with GFP-fused proteins, have emphasized the nonuniform nature of the wall. Insights are accumulating on the regulation of cell wall biosynthesis and composition 
under various conditions. Furthermore, evidence is emerging that the cell wall is a determinant for the establishment and maintenance of cellular polarity. In the future, these developments will connect with the other research on cell polarity and signal transduction to increase our understanding of the coordination of the response to internal and external changes with ongoing growth and proliferation.

\section{Acknowledgements}

We thank J. R. Pringle for his useful comments on the manuscript. We want to apologize to the many authors whose work has not been cited. Due to space limitations we have limited ourselves mainly to publications that have appeared in the past few years.

\section{References}

Alberts, A. S., Bouquin, N., Johnston, L. H. \& Treisman, R. (1998). Analysis of RhoA-binding proteins reveals an interaction domain conserved in heterotrimeric $\mathrm{G}$ protein $\beta$ subunits and the yeast response regulator protein Skn7. J Biol Chem 273, 8616-8622.

Andrews, P. D. \& Stark, M. J. R. (2000). Dynamic, Rho1pdependent localization of $\mathrm{Pkc1p}$ to sites of polarized growth. $J$ Cell Sci 113, 2685-2693.

Ayscough, K. R., Eby, J. J., Lila, T., Dewar, H., Kozminski, K. G. \& Drubin, D. G. (1999). Sla1p is a functionally modular component of the yeast cortical actin cytoskeleton required for correct localization of both Rho1p-GTPase and Sla2p, a protein with talin homology. Mol Biol Cell 10, 1061-1075.

Banuett, F. (1998). Signalling in the yeasts: an informational cascade with links to the filamentous fungi. Microbiol Mol Biol Rev 62, 249-274.

Barbet, N. C., Schneider, U., Helliwell, S. B., Stansfield, I., Tuite, M. F. \& Hall, M. N. (1996). TOR controls translation initiation and early G1 progression in yeast. Mol Biol Cell 7, 25-42.

Bickle, M., Delley, P. A., Schmidt, A. \& Hall, M. N. (1998). Cell wall integrity modulates RHO1 activity via the exchange factor ROM2. EMBO J 17, 2235-2245.

Cabib, E., Drgon, T., Drgonová, J., Ford, R. A. \& Kollár, R. (1997). The yeast cell wall, a dynamic structure engaged in growth and morphogenesis. Biochem Soc Trans 25, 200-204.

Cabib, E., Drgonová, J. \& Drgon, T. (1998). Role of small G proteins in yeast cell polarization and wall biosynthesis. Annu Rev Biochem 67, 307-333.

Cappellaro, C., Mrsa, V. \& Tanner, W. (1998). New potential cell wall glucanases of Saccharomyces cerevisiae and their involvement in mating. J Bacteriol 180, 5030-5037.

Chaffin, W. L., Lopez-Ribot, J. L., Casanova, M., Gozalbo, D. \& Martinez, J. P. (1998). Cell wall and secreted proteins of Candida albicans: identification, function, and expression. Microbiol Mol Biol Rev 62, 130-180.

Choi, W. J., Santos, B., Duran, A. \& Cabib, E. (1994a). Are yeast chitin synthases regulated at the transcriptional or the posttranslational level? Mol Cell Biol 14, 7685-7694.

Choi, W. J., Sburlati, A. \& Cabib, E. (1994b). Chitin synthase 3 from yeast has zymogenic properties that depend on both the CAL1 and the CAL3 genes. Proc Natl Acad Sci USA 91, 4727-4730.

Christodoulidou, A., Briza, P., Ellinger, A. \& Bouriotis, V. (1999).
Yeast ascospore wall assembly requires two chitin deacetylase isozymes. FEBS Lett 460, 275-279.

Chu, S., DeRisi, J., Eisen, M., Mulholland, J., Botstein, D., Brown, P. O. \& Herskowitz, I. (1998). The transcriptional program of sporulation in budding yeast. Science 282, 699-705.

Chuang, J. S. \& Schekman, R. W. (1996). Differential trafficking and timed localization of two chitin synthase proteins, Chs $2 \mathrm{p}$ and Chs3p. J Cell Biol 135, 597-610.

Delley, P. A. \& Hall, M. N. (1999). Cell wall stress depolarizes cell growth via hyperactivation of RHO1. J Cell Biol 147, 163-174.

DeMarini, D. J., Adams, A. E., Fares, H., De Virgilio, C., Valle, G., Chuang, J. S. \& Pringle, J. R. (1997). A septin-based hierarchy of proteins required for localized deposition of chitin in the Saccharomyces cerevisiae cell wall. J Cell Biol 139, 75-93.

De Nobel, J. G., Ruiz, C., Martin, H., Morris, W., Brul, S., Molina, M. \& Klis, F. M. (2000). Cell wall perturbation in yeast results in dual phosphorylation of the Slt2/Mpk1 MAP kinase and in an Slt2-mediated increase in FKS2-lacZ expression, glucanase resistance and thermotolerance. Microbiology 146, 2121-2132.

Di Como, C. J. \& Arndt, K. T. (1996). Nutrients, via the Tor proteins, stimulate the association of Tap42 with type 2A phosphatases. Genes Dev 10, 1904-1916.

Dodou, E. \& Treisman, R. (1997). The Saccharomyces cerevisiae MADS-box transcription factor Rlm1 is a target for the Mpk1 mitogen-activated protein kinase pathway. Mol Cell Biol 17, 1848-1859.

Drgonová, J., Drgon, T., Roh, D. H. \& Cabib, E. (1999). The GTPbinding protein Rho1p is required for cell cycle progression and polarization of the yeast cell. J Cell Biol 146, 373-387.

Erdman, S., Lin, L., Malczynski, M. \& Snyder, M. (1998). Pheromone-regulated genes required for yeast mating differentiation. J Cell Biol 140, 461-483.

Gammie, A. E., Brizzio, V. \& Rose, M. D. (1998). Distinct morphological phenotypes of cell fusion mutants. Mol Biol Cell 9, 1395-1410.

Garcia-Rodriguez, L. J., Trilla, J. A., Castro, C., Valdivieso, M. H., Duran, A. \& Roncero, C. (2000). Characterization of the chitin biosynthesis process as a compensatory $\mathrm{F}$ mechanism in the fks1 mutant of Saccharomyces cerevisiae. FEBS Lett 478, 84-88.

Goldman, R. C., Sullivan, P. A., Zakula, D. \& Capobianco, J. O. (1995). Kinetics of $\beta-1,3$ glucan interaction at the donor and acceptor sites of the fungal glucosyltransferase encoded by the BGL2 gene. Eur J Biochem 227, 372-378.

Gray, J. V., Ogas, J. P., Kamada, Y., Stone, M., Levin, D. E. \& Herskowitz, I. (1997). A role for the Pkc1 MAP kinase pathway of Saccharomyces cerevisiae in bud emergence and identification of a putative upstream regulator. EMBO J 16, 4924-4937.

Gustin, M. C., Albertyn, J., Alexander, M. \& Davenport, K. (1998). MAP kinase pathways in the yeast Saccharomyces cerevisiae. Microbiol Mol Biol Rev 62, 1264-1300.

Helliwell, S. B., Howald, I., Barbet, N. \& Hall, M. N. (1998a). TOR2 is part of two related signaling pathways coordinating cell growth in Saccharomyces cerevisiae. Genetics 148, 99-112.

Helliwell, S. B., Schmidt, A., Ohya, Y. \& Hall, M. N. (1998b). The Rho1 effector Pkc1, but not Bni1, mediates signaling from Tor2 to the actin cytoskeleton. Curr Biol 8, 1211-1214.

Igual, J. C., Johnson, A. L. \& Johnston, L. H. (1996). Coordinated regulation of gene expression by the cell cycle transcription factor Swi4 and the protein kinase C MAP kinase pathway for yeast cell integrity. EMBO J 15, 5001-5013.

Imamura, H., Tanaka, K., Hihara, T., Umikawa, M., Kamei, T., 
Takahashi, K., Sasaki, T. \& Takai, Y. (1997). Bni1p and Bnr1p: downstream targets of the Rho family small G-proteins which interact with profilin and regulate actin cytoskeleton in Saccharomyces cerevisiae. EMBO J 16, 2745-2755.

Inoue, S. B., Qadota, H., Arisawa, M., Watanabe, T. \& Ohya, Y. (1999). Prenylation of Rho1p is required for activation of yeast 1,3- $\beta$-glucan synthase. J Biol Chem 274, 38119-38124.

Ivanovska, I. \& Rose, M. D. (2000). SLG1 plays a role during G1 in the decision to enter or exit the cell cycle. Mol Gen Genet 262, 1147-1156.

Jacoby, J. J., Nilius, S. M. \& Heinisch, J. J. (1998). A screen for upstream components of the yeast protein kinase $\mathrm{C}$ signal transduction pathway identifies the product of the $S L G 1$ gene. Mol Gen Genet 258, 148-155.

Jung, U. S. \& Levin, D. E. (1999). Genome-wide analysis of gene expression regulated by the yeast cell wall integrity signaling pathway. Mol Microbiol 34, 1049-1057.

Kapteyn, J. C., Van Den Ende, H. \& Klis, F. M. (1999a). The contribution of cell wall proteins to the organization of the yeast cell wall. Biochim Biophys Acta 1426, 373-383.

Kapteyn, J. C., Van Egmond, P., Sievi, E., Van Den Ende, H., Makarow, M. \& Klis, F. M. (1999b). The contribution of the $O-$ glycosylated protein Pir2p/Hsp150 to the construction of the yeast cell wall in wild-type cells and $\beta$ 1,6-glucan-deficient mutants. Mol Microbiol 31, 1835-1844.

Kapteyn, J. C., Hoyer, L. L., Hecht, J. E., Muller, W. H., Andel, A., Verkleij, A. J., Makarow, M., Van Den Ende, H. \& Klis, F. M. (2000). The cell wall architecture of Candida albicans wild-type cells and cell wall-defective mutants. Mol Microbiol 35, 601-611.

Ketela, T., Green, R. \& Bussey, H. (1999). Saccharomyces cerevisiae Mid2p is a potential cell wall stress sensor and upstream activator of the PKC1-MPK1 cell integrity pathway. J Bacteriol 181, 3330-3340.

Kim, Y. J., Francisco, L., Chen, G. C., Marcotte, E. \& Chan, C. S. (1994). Control of cellular morphogenesis by the Ip12/Bem2 GTPase-activating protein: possible role of protein phosphorylation. J Cell Biol 127, 1381-1394.

King, L. \& Butler, G. (1998). Ace2p, a regulator of CTS1 (chitinase) expression, affects pseudohyphal production in Saccharomyces cerevisiae. Curr Genet 34, 183-191.

Klis, F. M. (1994). Review : cell wall assembly in yeast. Yeast 10, 851-869.

Kohno, H., Tanaka, K., Mino, A. \& 9 other authors (1996). Bnilp implicated in cytoskeletal control is a putative target of Rho1p small GTP binding protein in Saccharomyces cerevisiae. EMBO J 15, 6060-6068.

Kovacech, B., Nasmyth, K. \& Schuster, T. (1996). EGT2 gene transcription is induced predominantly by Swi5 in early G1. Mol Cell Biol 16, 3264-3274.

Kubler, E., Mosch, H. U., Rupp, S. \& Lisanti, M. P. (1997). Gpa2p, a G-protein $\alpha$-subunit, regulates growth and pseudohyphal development in Saccharomyces cerevisiae via a cAMP-dependent mechanism. J Biol Chem 272, 20321-20323.

Kupiec, M., Byers, B., Esposito, R. E. \& Mitchell, A. P. (1997). Meiosis and sporulation in Saccharomyces cerevisiae. In The Molecular Biology of the Yeast Saccharomyces, vol. 3, pp. 889-1036. Edited by J. R. Pringle, J. R. Broach \& E. W. Jones. Cold Spring Harbor, NY: Cold Spring Harbor Laboratory.

Lambrechts, M. G., Bauer, F. F., Marmur, J. \& Pretorius, I. S. (1996). Muc1, a mucin-like protein that is regulated by Mss10, is critical for pseudohyphal differentiation in yeast. Proc Natl Acad Sci US A 93, 8419-8424.
Lo, H. J., Kohler, J. R., DiDomenico, B., Loebenberg, D., Cacciapuoti, A. \& Fink, G. R. (1997). Nonfilamentous C. albicans mutants are avirulent. Cell 90, 939-949.

Lo, W. S. \& Dranginis, A. M. (1998). The cell surface flocculin Flo11 is required for pseudohyphae formation and invasion by Saccharomyces cerevisiae. Mol Biol Cell 9, 161-171.

Longtine, M. S., DeMarini, D. J., Valencik, M. L., Al-Awar, O. S., Fares, H., De Virgilio, C. \& Pringle, J. R. (1996). The septins: roles in cytokinesis and other processes. Curr Opin Cell Biol 8, 106-119.

Longtine, M. S., Fares, H. \& Pringle, J. R. (1998). Role of the yeast Gin $4 p$ protein kinase in septin assembly and the relationship between septin assembly and septin function. J Cell Biol 143, 719-736.

Lorenz, M. C. \& Heitman, J. (1997). Yeast pseudohyphal growth is regulated by GPA2, a $G$ protein $\alpha$ homolog. EMBO J 16, 7008-7018.

Marsh, L. \& Rose, M. D. (1997). The pathway of cell and nuclear fusion during mating in S. cerevisiae. In The Molecular Biology of the Yeast Saccharomyces, vol. 3, pp. 827-888. Edited by J. R. Pringle, J. R. Broach \& E. W. Jones. Cold Spring Harbor, NY: Cold Spring Harbor Laboratory.

Martin, H., Rodriguez-Pachon, J. M., Ruiz, C., Nombela, C. \& Molina, M. (2000). Regulatory mechanisms for modulation of signaling through the cell integrity Slt2-mediated pathway in Saccharomyces cerevisiae. J Biol Chem 275, 1511-1519.

Mazur, P., Morin, N., Baginsky, W., el-Sherbeini, M., Clemas, J. A., Nielsen, J. B. \& Foor, F. (1995). Differential expression and function of two homologous subunits of yeast $1,3-\beta$-D-glucan synthase. Mol Cell Biol 15, 5671-5681.

Mosch, H. U., Roberts, R. L. \& Fink, G. R. (1996). Ras2 signals via the $\mathrm{Cdc} 42 / \mathrm{Ste} 20 /$ mitogen-activated protein kinase module to induce filamentous growth in Saccharomyces cerevisiae. Proc Natl Acad Sci U S A 93, 5352-5356.

Mouyna, I., Fontaine, T., Vai, M., Monod, M., Fonzi, W. A., Diaquin, M., Popolo, L., Hartland, R. P. \& Latge, J. P. (2000). Glycosylphosphatidylinositol-anchored glucanosyltransferases play an active role in the biosynthesis of the fungal cell wall. $J$ Biol Chem 275, 14882-14889.

Ono, N., Yabe, T., Sudoh, M., Nakajima, T., Yamada-Okabe, T., Arisawa, M. \& Yamada-Okabe, H. (2000). The yeast Chs4 protein stimulates the trypsin-sensitive activity of chitin synthase 3 through an apparent protein-protein interaction. Microbiology 146, 385-391.

Ono, T., Suzuki, T., Anraku, Y. \& lida, H. (1994). The MID2 gene encodes a putative integral membrane protein with a $\mathrm{Ca}^{2+}$ binding domain and shows mating pheromone-stimulated expression in Saccharomyces cerevisiae. Gene 151, 203-208.

Orlean, P. (1997). Biogenesis of yeast wall and surface components. In The Molecular Biology of the Yeast Saccharomyces, vol. 3, pp. 229-362. Edited by J. R. Pringle, J. R. Broach \& E. W. Jones. Cold Spring Harbor, NY: Cold Spring Harbor Laboratory.

Osmond, B. C., Specht, C. A. \& Robbins, P. W. (1999). Chitin synthase III: synthetic lethal mutants and 'stress related' chitin synthesis that bypasses the CSD3/CHS6 localization pathway. Proc Natl Acad Sci US A 96, 11206-11210.

Ozaki, K., Tanaka, K., Imamura, H., Hihara, T., Kameyama, T., Nonaka, H., Hirano, H., Matsuura, Y. \& Takai, Y. (1996). Rom1p and Rom $2 p$ are GDP/GTP exchange proteins (GEPs) for the Rho1p small GTP binding protein in Saccharomyces cerevisiae. EMBO J 15, 2196-2207.

Pan, X. \& Heitman, J. (1999). Cyclic AMP-dependent protein 
kinase regulates pseudohyphal differentiation in Saccharomyces cerevisiae. Mol Cell Biol 19, 4874-4887.

Rajavel, M., Philip, B., Buehrer, B. M., Errede, B. \& Levin, D. E. (1999). Mid2 is a putative sensor for cell integrity signaling in Saccharomyces cerevisiae. Mol Cell Biol 19, 3969-3976.

Reif, K., Nobes, C. D., Thomas, G., Hall, A. \& Cantrell, D. A. (1996). Phosphatidylinositol 3-kinase signals activate a selective subset of Rac/Rho-dependent effector pathways. Curr Biol 6, 1445-1455.

Robertson, L. S. \& Fink, G. R. (1998). The three yeast A kinases have specific signaling functions in pseudohyphal growth. Proc Natl Acad Sci U S A 95, 13783-13787.

Rodriguez-Peña, J. M., Cid, V. J., Arroyo, J. \& Nombela, C. (2000). A novel family of cell wall-related proteins regulated differently during the yeast life cycle. Mol Cell Biol 20, 3245-3255.

Rupp, S., Summers, E., Lo, H. J., Madhani, H. \& Fink, G. (1999). MAP kinase and cAMP filamentation signaling pathways converge on the unusually large promoter of the yeast FLO11 gene. EMBO J 18, 1257-1269.

Santos, B. \& Snyder, M. (1997). Targeting of chitin synthase 3 to polarized growth sites in yeast requires Chs5p and Myo2p. J Cell Biol 136, 95-110.

Santos, B., Duran, A. \& Valdivieso, M. H. (1997). CHS5, a gene involved in chitin synthesis and mating in Saccharomyces cerevisiae. Mol Cell Biol 17, 2485-2496.

Schmidt, A., Kunz, J. \& Hall, M. N. (1996). TOR2 is required for organization of the actin cytoskeleton in yeast. Proc Natl Acad Sci US A 93, 13780-13785.

Schmidt, A., Bickle, M., Beck, T. \& Hall, M. N. (1997). The yeast phosphatidylinositol kinase homolog TOR2 activates RHO1 and RHO2 via the exchange factor ROM2. Cell 88, 531-542.

Smits, G. J., Kapteyn, J. C., Van Den Ende, H. \& Klis, F. M. (1999). Cell wall dynamics in yeast. Curr Opin Microbiol 2, 348-352.

Spellman, P. T., Sherlock, G., Zhang, M. Q., lyer, V. R., Anders, K., Eisen, M. B., Brown, P. O., Botstein, D. \& Futcher, B. (1998). Comprehensive identification of cell cycle-regulated genes of the yeast Saccharomyces cerevisiae by microarray hybridization. Mol Biol Cell 9, 3273-3297.

Trilla, J. A., Cos, T., Duran, A. \& Roncero, C. (1997). Characterization of CHS4 (CAL2), a gene of Saccharomyces cerevisiae involved in chitin biosynthesis and allelic to SKT5 and CSD4. Yeast 13, 795-807.

Trilla, J. A., Duran, A. \& Roncero, C. (1999). Chs7p, a new protein involved in the control of protein export from the endoplasmic reticulum that is specifically engaged in the regulation of chitin synthesis in Saccharomyces cerevisiae. J Cell Biol 145, 1153-1163.

Valdivieso, M. H., Ferrario, L., Vai, M., Duran, A. \& Popolo, L. (2000). Chitin synthesis in a gas1 mutant of Saccharomyces cerevisiae. J Bacteriol 182, 4752-4757.

Verna, J., Lodder, A., Lee, K., Vagts, A. \& Ballester, R. (1997). A family of genes required for maintenance of cell wall integrity and for the stress response in Saccharomyces cerevisiae. Proc Natl Acad Sci U S A 94, 13804-13809.

Wagner, M., Briza, P., Pierce, M. \& Winter, E. (1999). Distinct steps in yeast spore morphogenesis require distinct SMK1 MAP kinase thresholds. Genetics 151, 1327-1340.

Watanabe, Y., Takaesu, G., Hagiwara, M., Irie, K. \& Matsumoto, K. (1997). Characterization of a serum response factor-like protein in Saccharomyces cerevisiae, Rlm1, which has transcriptional activity regulated by the Mpk1 (Slt2) mitogen-activated protein kinase pathway. Mol Cell Biol 17, 2615-2623.

Zhao, C., Jung, U. S., Garrett-Engele, P., Roe, T., Cyert, M. S. \& Levin, D. E. (1998). Temperature-induced expression of yeast $F K S 2$ is under the dual control of protein kinase $\mathrm{C}$ and calcineurin. Mol Cell Biol 18, 1013-1022.

Ziman, M., Chuang, J. S. \& Schekman, R. W. (1996). Chs1p and Chs3p, two proteins involved in chitin synthesis, populate a compartment of the Saccharomyces cerevisiae endocytic pathway. Mol Biol Cell 7, 1909-1919.

Ziman, M., Chuang, J. S., Tsung, M., Hamamoto, S. \& Schekman, R. (1998). Chs6p-dependent anterograde transport of Chs3p from the chitosome to the plasma membrane in Saccharomyces cerevisiae. Mol Biol Cell 9, 1565-1576. 